\title{
Enhancement of tensile properties of surface treated oil palm mesocarp fiber/poly(butylene succinate) biocomposite by (3-aminopropyl)trimethoxysilane
}

\begin{abstract}
The issue related to relatively poor interfacial adhesion between hydrophilic natural fiber and hydrophobic thermoplastic remain as an obstacle in natural fiber/thermoplastic biocomposites. Consequently, surface treatment of fiber is of important to impart adhesion. The present work used consecutive superheated steam-alkali treatment to treat the oil palm mesocarp fiber (OPMF) prior to biocomposite fabrication. The biocomposites made up of 70 wt $\%$ treated OPMF and $30 \mathrm{wt} \%$ poly (butylene succinate) (PBS) were prepared by melt blending technique in a Brabender internal mixer followed by hot-press moulding into $1 \mathrm{~mm}$ sheets. A silane coupling agent of (3-aminopropyl) trimethoxysilane (APTMS) was also added to the biocomposite during the process of compounding to promote interfacial adhesion and enhance the properties of biocomposites. The results showed that the biocomposite containing $2 \mathrm{wt} \%$ APTMS showed maximum enhancement in tensile strength $(89 \%)$, tensile modulus $(812 \%)$ and elongation at break (52\%) in comparison to that of untreated OPMF/PBS biocomposite. The SEM observation of the tensile fracture surface revealed that APTMS improved the interfacial adhesion between treated OPMF and PBS. It can be deduced that the presence of APTMS can improve the adhesion between hydrophilic fiber and hydrophobic thermoplastic, and thus increased the tensile properties of the biocomposite.
\end{abstract}

Keyword: (3-aminopropyl)trimethoxysilane; Biocomposites; Oil palm mesocarp fiber; Poly(butylene succinate); Tensile properties 